\section{VLSI DESIGN}

1996, Vol. 4, No. 1, pp. 75-81

Reprints available directly from the publisher

Photocopying permitted by license only
C 1996 OPA (Overseas Publishers Association) Amsterdam B.V.

Published in The Netherlands under license by Gordon and Breach Science Publishers SA

Printed in Malaysia

\title{
Design and Implementation of a Low Power Ternary Full Adder
}

\author{
A. SRIVASTAVA and K. VENKATAPATHY \\ Department of Electrical and Computer Engineering, Louisiana State University, Baton Rouge, LA 70803-5901 \\ Phone: (504) 388-5622 Fax: (504) 388-5622 Email: ashok@gate.ee.lsu.edu
}

(Received November 29, 1993, Revised April 26, 1995)

\begin{abstract}
In this work, the design and implementation of a low power ternary full adder are presented in CMOS technology. In a ternary full adder design, the basic building blocks, the positive ternary inverter (PTI) and negative ternary inverter (NTI) are developed using a CMOS inverter and pass transistors. In designs of PTI and NTI, W/L ratios of transistors have been varied for their optimum performance. The ternary full adder and its building blocks have been simulated with SPICE 2G.6 using the MOSIS model parameters. The rise and fall times of PTI show an improvement by a factor of 14 and 4 , respectively, and that of the NTI by a factor of nearly 4 and 17, respectively over that of earlier designs implemented in depletion-enhancement CMOS (DECMOS) technology. The noise margins improve by a factor of nearly 2 in PTI and NTI, respectively.

The ternary full adder has been fabricated in MOSIS two micron n-well CMOS technology. The full adder and its building blocks, NTI and PTI have been tested experimentally for static and dynamic performance, compared with the SPICE simulated behavior, and close agreement is observed.

The ternary-valued logic circuits designed in the present work which do not use depletion mode MOSFETS perform better than that implemented earlier in DECMOS technology. The present design is fully compatible with the current CMOS technology, uses fewer components and dissipates power in the microwatt range.
\end{abstract}

Key Words: CMOS Ternary Full Adder, Ternary Logic, 3-Valued Logic, Low Power CMOS Full Adder

\section{INTRODUCTION}

$\mathbf{T}$ The performance of two levels (binary logic) is limited due to interconnect which occupies large area on a VLSI chip. In a VLSI circuit, approximately 70 percent of the area is devoted to interconnection, 20 percent to insulation, and 10 percent to devices [1]. One can achieve a more cost effective way of utilizing interconnections by using a larger set of signals over the same area in multiple-valued logic (MVL) circuits. This also solves the problem of pinout (the limit to the amount of data that can enter and exit a chip). Commercially multiple-valued logic circuits have made an appearance with the four-valued read-only memory (ROM) which Intel used in the control store of its 8087 numeric coprocessor [1]. Hitachi has introduced into the market a 16-valued mass memory with a high storage capacity. Kameyama et al. [2] reported a $32 \times 32$ bit signed digit (SD) multiplier implementation using MVL circuits realized in current-mode CMOS technology. The chip area and power dissipation of MVL multiplier implementa- tion reduced to half that of the fastest conventional binary realization of the same multiplier.

The main draw back in multiple valued logic circuits is that their design techniques are more complex than the binary logic circuits [3]. The implementation of MVL circuits have ranged through integrated injection logic, emitter coupled logic, CMOS and n-MOS technologies and charge-coupled devices. In this work, the design of ternary-valued logic circuits have been explored over other ternary-valued logic due to the following reasoning. In a numerical system, the number $\mathrm{N}$ is given by $\mathrm{N}=\mathrm{R}^{\mathrm{d}}$ where $R$ is the radix and $d$ is the necessary number of digits up to the next highest integer value where necessary. If the cost or complexity $C$ in any system is assumed to be proportional to $\mathrm{R} \times \mathrm{D}$ [4], then $C=k(R \times d)=k[\mathrm{R}(\ln \mathrm{N} / \mathrm{ln} \mathrm{R})]$ where $k$ is some constant. Differentiating with respect to $R$ will show that for a minimum cost $C, R$ should be equal to $e(2.718)$. Since in practice $R$ must be an integer, this suggests that $R=3$ (ternary) would be more economical than $R=$ 2(binary) [4]. 
Several authors [5-9] have used CMOS integrated circuits for the realization of three-valued logic circuits. These designs have used power supply voltages higher than the MOSFETs threshold voltage which resulted in high power consumption in the circuits. Mouftah and Smith [10] have reported a family of low-power threevalued CMOS circuits. In order to further reduce the power dissipation, increase the speed and eliminate the use of linear resistors in these circuits, Heung and Mouftah [11] proposed a design of ternary logic circuits based on the use of depletion enhancement complementary metal-oxide-semiconductor (DECMOS) technology. However, their implementation is not compatible with the current CMOS technology. The present CMOS technology does not use depletion mode transistors. The prime objective in our work is to minimize the number of transistors used, eliminate the use of resistors to lower the power consumption, reduce the propagation delay time and eliminate depletion mode transistors. The reduction in the number of transistors is our main focus as that enabled a more compact design which utilized the less chip area. The designs of positive ternary inverter (PTI), negative ternary inverter (NTI) and simple ternary inverter (STI) are based on use of a CMOS inverter and pass transistors/CMOS transmission gate at its output. The pass transistors at the output of inverter have been used to pull the output node to the required voltage levels and also provide sufficient equivalent resistance for the ternary logic implementation. The two unary operators PTI and NTI have been used to design a $J_{k}$ arithmetic circuit, and ternary gate (T-gate) which is essentially a multiplexer. Fourteen such T-gates have been finally used in design of a ternary full adder. The design has been fabricated in MOSIS two micron CMOS n-well process, tested and performance verified.

\section{DESIGN OF CMOS 3-VALUED LOGIC CIRCUITS}

Three types of basic ternary operations are defined by [11]

$$
\bar{X}_{C}=\left\{\begin{array}{cc}
C & \text { if } X=1 \\
2-X & \text { if } X \neq 1
\end{array}\right.
$$

C in Eq. (1) takes the values of logic 2 for a PTI, logic 1 for a STI and logic 0 for a NTI which correspond to higher level (1), middle level (0) and lower level (-1), respectively.

Fig. 1 shows the schematic of a positive ternary inverter (PTI). A p-MOSFET $\left(\mathrm{Q}_{3}\right)$ is connected to the output of a standard CMOS inverter. Mouftah and Garba [12] have pointed out that by altering the length-to-width ratio of the PMOS and NMOS channels can significantly

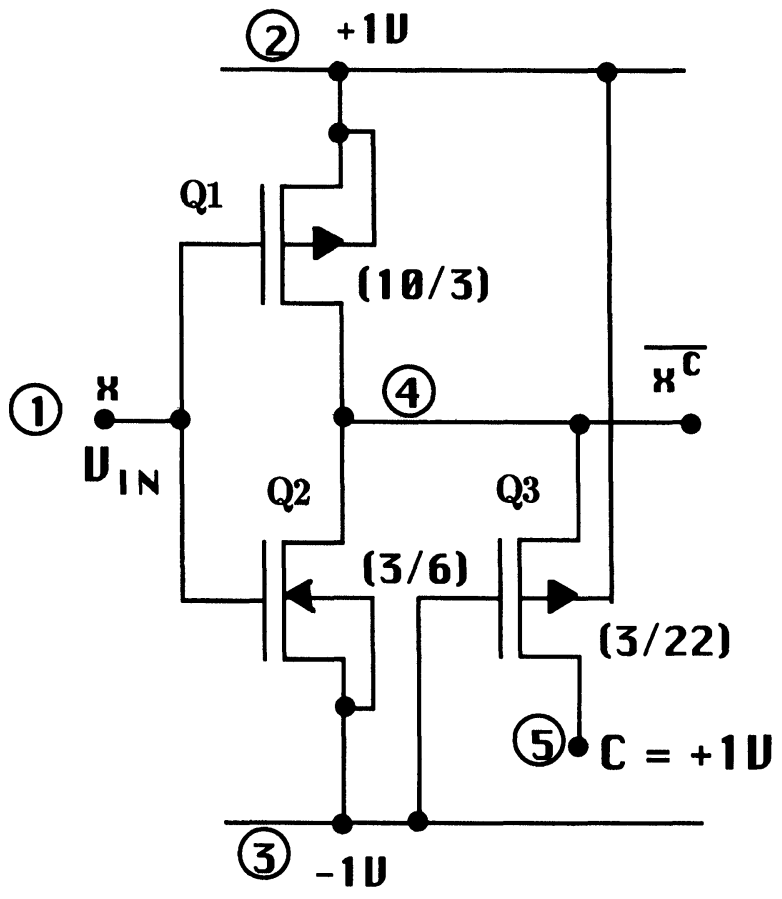

FIGURE 1 Positive ternary inverter.

change the resistance of channels. Thus, the resistance of the circuit is directly proportional to its $\mathrm{L} / \mathrm{W}$ ratio which can be effectively used to change the resistance of transistors to suit design needs. However, there is a lower limit to the value of $\mathrm{L}$ and $\mathrm{W}$ due to the limitations imposed by the design rules of the foundry which in the present case is W/L of $3 / 2$. In Fig. 1 the gate of p-MOSFET $\left(Q_{3}\right)$ has been tied to the negative power supply to keep it constantly turned on. A control signal, $\mathrm{C}$ of $+1 \mathrm{~V}$ is applied to the source of p-MOSFET $\left(\mathrm{Q}_{3}\right)$. The $W / L$ ratio of $p$ - and $n$-MOSFETs $\left(Q_{1}\right.$ and $\left.Q_{2}\right)$ in CMOS inverter are $10 / 3$ and $3 / 6$, respectively, and that of p-MOSFET $\left(Q_{3}\right)$ connected to the output is $3 / 22$. The p-MOSFET $\left(\mathrm{Q}_{3}\right)$ pulls the output of the CMOS inverter to $+1 \mathrm{~V}$ during the cycle where both transistors of the inverter are nearly in cut-off.

Fig. 2 shows the schematic of a negative ternary inverter (NTI). An n-MOSFET $\left(\mathrm{Q}_{3}\right)$ is connected to the output of a CMOS inverter with its gate tied to the positive power supply to keep it constantly turned on. A control signal, $C$ of $-1 \mathrm{~V}$ is applied to the source of $n$-MOSFET $\left(Q_{3}\right)$ and that pulls the output of CMOS inverter to that value. The CMOS inverter is forced to a value of $-1 \mathrm{~V}$ in phase where both transistors of the CMOS inverter are in the cut-off region. The $\mathrm{W} / \mathrm{L}$ ratio of p- and n-MOSFET $\left(\mathrm{Q}_{1}\right.$ and $\left.\mathrm{Q}_{2}\right)$ comprising the CMOS inverter are $19 / 3$ and $12 / 3$, respectively and that of $n$-MOSFET $\left(Q_{3}\right)$ connected to the output is $6 / 23$. The 


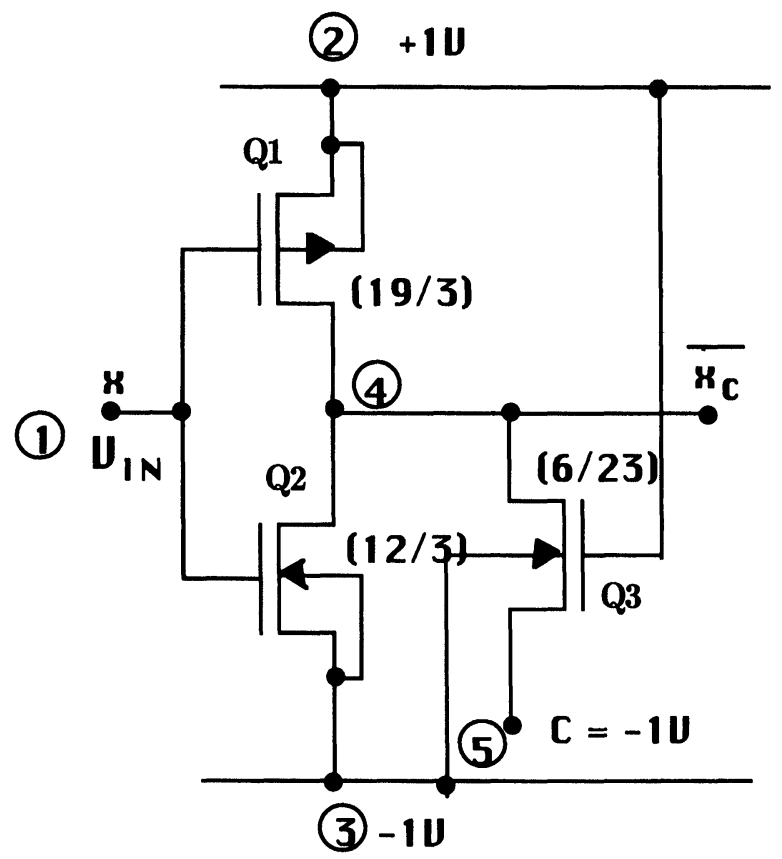

FIGURE 2 Negative ternary inverter.

value of $6 / 23$ was chosen for $\mathrm{W} / \mathrm{L}$ ratio so as to make it more resistive and avoid the pass transistor to latch the output of the whole circuit to $-1 \mathrm{~V}$.

Fig. 3 shows the schematic of a simple ternary inverter (STI) designed by connecting a CMOS transmission gate to the common drain output of a CMOS inverter. The gates of $\mathrm{p}$ - and $\mathrm{n}$ - MOSFETs $\left(\mathrm{Q}_{3}\right.$ and $\left.\mathrm{Q}_{4}\right)$ in the transmission gate are tied to negative and positive power supplies, respectively. The $\mathrm{W} / \mathrm{L}$ ratio of $\mathrm{p}-$ and $\mathrm{n}$ MOSFETs $\left(Q_{1}\right.$ and $\left.Q_{2}\right)$ are $77 / 3$ and $75 / 3$, respectively and the corresponding values of transistors $\left(\mathrm{Q}_{3}\right.$ and $\left.\mathrm{Q}_{4}\right)$ in transmission gate are $3 / 3$ for both. The transmission

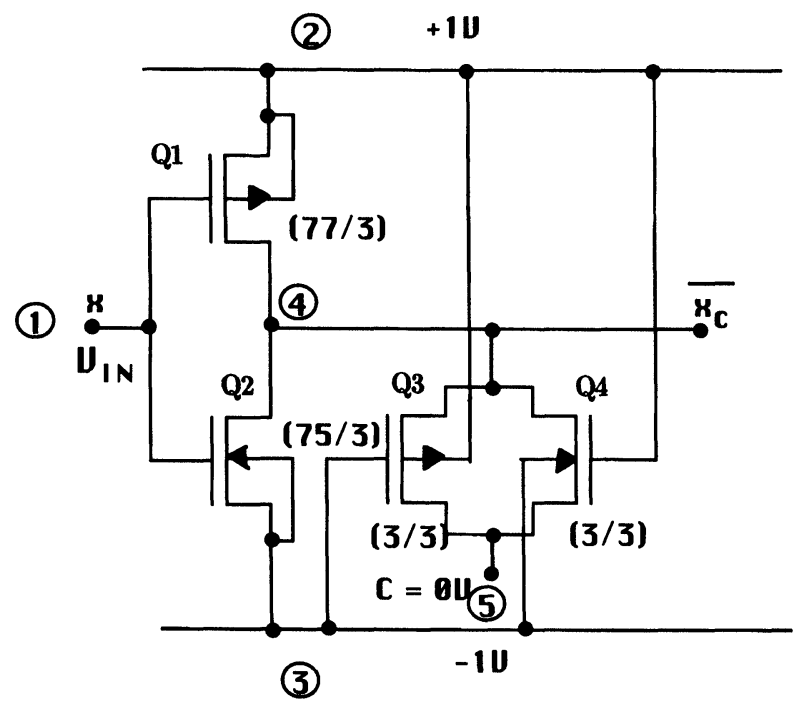

FIGURE 3 Simple ternary inverter. gate aids in pulling up a control signal, $C$ of $0 \mathrm{~V}$ to the output when the inverter is in cut-off.

Figures 4 and 5 show the circuits for ternary NAND and ternary NOR, respectively. They are designed by connecting a CMOS transmission gate to the common drain output of a binary CMOS NAND and NOR. The gates of $\mathrm{p}$ - and n-MOSFETs $\left(\mathrm{Q}_{5}\right.$ and $\left.\mathrm{Q}_{6}\right)$ in the transmission gate are tied to negative and positive power supplies, respectively. It can be seen from the Fig. 4 for ternary NAND that the transmission gate at the output helps pull the output to $0 \mathrm{~V}$ when transistors $\left(\mathrm{Q}_{1}, \mathrm{Q}_{2}, \mathrm{Q}_{3}\right.$ and $\left.\mathrm{Q}_{4}\right)$ are in cut-off. This happens in cases when inputs $\mathrm{X}=0, \mathrm{Y}=0 ; \mathrm{X}=0, \mathrm{Y}=1$; and $\mathrm{X}=1, \mathrm{Y}=0$, respectively. Similary operation of ternary NOR for the Fig. 5 can be explained. The output pulls to $0 \mathrm{~V}$ when $\mathrm{X}$ $=-1, \mathrm{Y}=0 ; \mathrm{X}=0, \mathrm{Y}=-1$; and $\mathrm{X}=0, \mathrm{Y}=0$, respectively.

\section{TERNARY FULL ADDER DESIGN}

A ternary full adder is a circuit that will add two trits and a previous carry trit, and generate a sum trit and a carry trit (a trit is equivalent of a bit in a binary system). It can be implemented by using two ternary half adders and a binary OR gate by analogy with the typical binary full adder. The advantage of multiple-valued carry ripple adders is in fact that the carry is always binary. Since the carry propagation makes up most of the delay in a carry ripple adder, this suggests that a multiple-valued adder could have a speed advantage over its binary counterpart because each digit carries more information than the binary case [13]. In the present design, the full adder is composed of fourteen T-gates which are essentially multiplexers. Each T-gate is further composed of a $J_{k}$

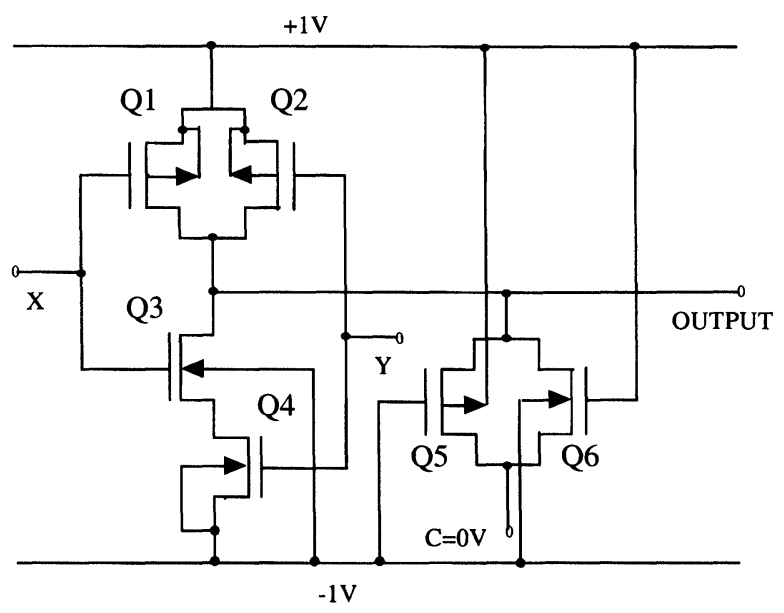

FIGURE 4 Ternary NAND circuit. 


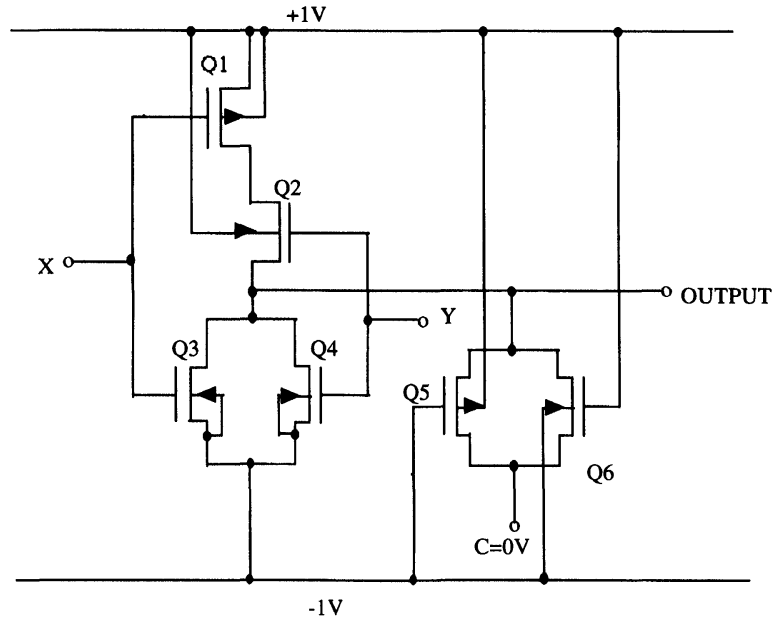

FIGURE 5 Ternary NOR circuit.

arithmetic circuit. The $J_{k}$ arithmetic function is defined by

$$
J_{k}(X)=\left\{\begin{array}{cc}
1 & \text { if } X=1 \\
-1 & \text { if } X \neq k
\end{array}\right.
$$

where $k$ can take values of logic 0 , logic 1 and logic 2 which corresponds to higher level (1), middle level (0) and lower level $(-1)$, respectively. The block diagram of a $J_{k}$ arithmetic circuit is shown in Fig. 6 which uses the logic design described in Ref. 13. The design of the T-gate circuit is based on the $J_{k}$ arithmetic circuit. The function of the T-gate is described as follows [11]

$$
T\left(y_{1}, y_{2}, y_{3} ; X\right)=y_{i}
$$

where $i$ will take a value of 1 if $X$ takes the value of -1 , 2 if $X$ is 0 , and 3 if $X$ is 1 . The block diagram of a T-gate is shown in Fig. 7. Each ternary switch consists of a p-channel and n-channel enhancement transistor. The source of p-channel MOSFET is connected to the drain of $n$-channel MOSFET and vice versa. A control signal, $C$ controls the n-channel MOSFET directly, and the p-channel MOSFET is controlled by $\bar{C}$. When $C$ is equal to $+1 \mathrm{~V}$ the switch will be on, for $\mathrm{C}$ equal to $-1 \mathrm{~V}$ the switch will be off. The $J_{-1}, J_{0}, J_{1}$ signals of the $J_{k}$ arithmetic circuits are connected to $C$ of the ternary

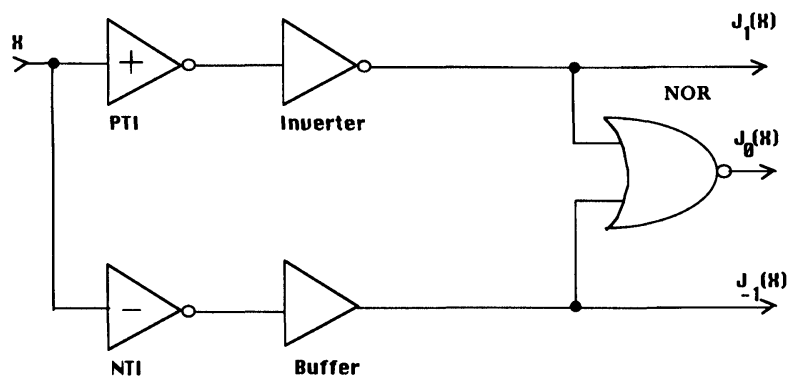

FIGURE 6 Block diagram of a $J_{k}$ arithmetic circuit. switch that has inputs $y_{1}, y_{2}, y_{3}$, respectively. The value of input to the $J_{k}$ arithmetic circuit determines which one of the signals $\left(y_{1}, y_{2}, y_{3}\right)$ will be steered to the output thus functioning as a multiplexer. The full adder comprises of fourteen T-gates as shown in Fig. 8. Since the $J_{k}$ arithmetic circuit part of the T-gate is common, we can effectively reduce the component count by making it common for three stages. The area occupied by the ternary adder as a whole can be conserved in this way. The complete ternary full adder has been simulated using SPICE 2G.6 and the corresponding truth table is summarized in Table 1.

\section{DESIGN VERIFICATION AND DISCUSSION}

The design was fabricated in MOSIS two micron CMOS $\mathrm{n}$-well process. The static and dynamic performance of the device were studied experimentally and compared with the corresponding SPICE 2G.6 simulation. Averaged Level 2 MOSFET model parameters from MOSIS were used and are summarized in Tables 2 and 3, respectively.

Figures 9(a) and (b) show the voltage transfer characteristics of PTI and NTI obtained from SPICE 2G.6 simulation, measurements, and Ref. 11, respectively. It can be seen from Figs. 9(a) and (b) that measured PTI and NTI characteristics have close agreements with the corresponding SPICE 2G.6 simulation. The present design of PTI and NTI also exhibit sharper voltage transfer characteristics compared to designs in Ref. 11.

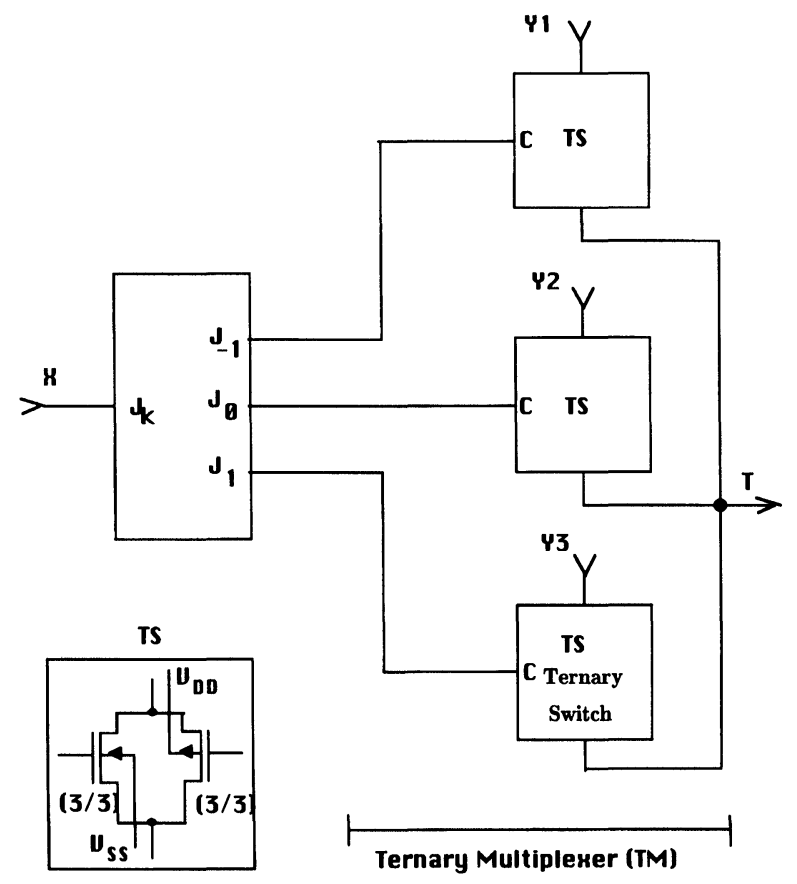

FIGURE 7 A ternary T-gate. 


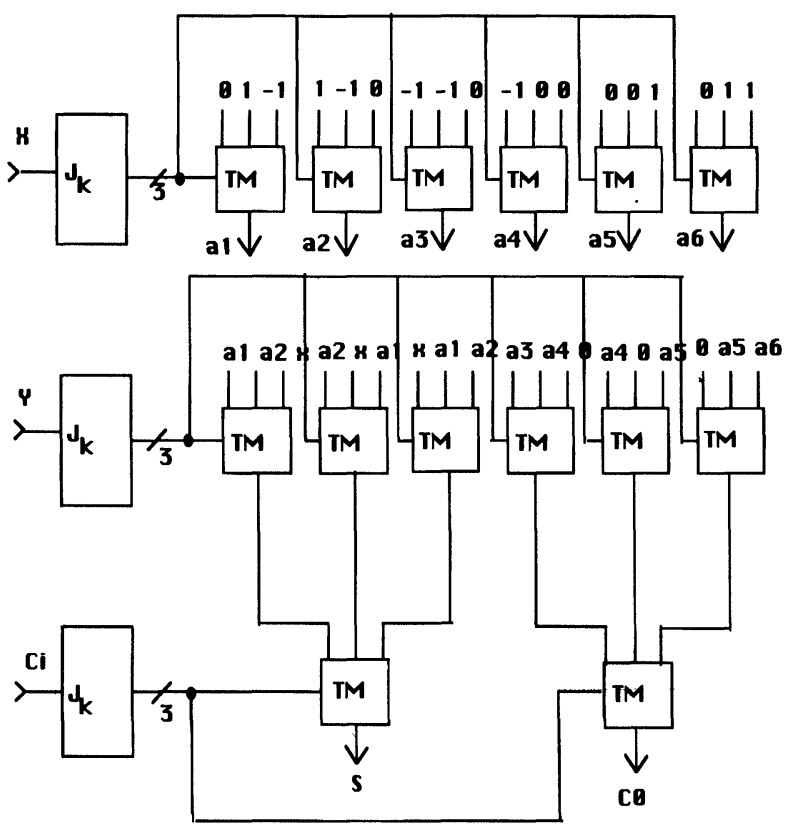

FIGURE 8 A ternary full adder.

TABLE 1

Truth table of a ternary full adder derived from SPICE simulation

\begin{tabular}{|c|c|c|c|c|}
\hline $\mathbf{x}$ & $y$ & $\mathrm{c}_{\mathrm{i}}$ & $\mathrm{s}$ & $\mathrm{c}_{\mathrm{o}}$ \\
\hline-1 & -1 & -1 & 0 & -1 \\
\hline-1 & -1 & 0 & 1 & -1 \\
\hline-1 & -1 & 1 & -1 & 0 \\
\hline-1 & 0 & -1 & 1 & -1 \\
\hline-1 & 0 & 0 & -1 & 0 \\
\hline-1 & 0 & 1 & 0 & 0 \\
\hline-1 & 1 & -1 & -1 & 0 \\
\hline-1 & 1 & 0 & 0 & 0 \\
\hline-1 & 1 & 1 & 1 & 0 \\
\hline 0 & -1 & -1 & 1 & -1 \\
\hline 0 & -1 & 0 & -1 & 0 \\
\hline 0 & -1 & 1 & 0 & 0 \\
\hline 0 & 0 & -1 & -1 & 0 \\
\hline 0 & 0 & 0 & 0 & 0 \\
\hline 0 & 0 & 1 & 1 & 0 \\
\hline 0 & 1 & -1 & 0 & -0 \\
\hline 0 & 1 & 0 & 1 & 0 \\
\hline 0 & 1 & 1 & -1 & 1 \\
\hline 1 & -1 & -1 & -1 & 0 \\
\hline 1 & -1 & 0 & 0 & 0 \\
\hline 1 & -1 & 1 & 1 & 0 \\
\hline 1 & 0 & -1 & 0 & 0 \\
\hline 1 & 0 & 0 & 1 & 0 \\
\hline 1 & 0 & 1 & -1 & 1 \\
\hline 1 & 1 & -1 & 1 & 0 \\
\hline 1 & 1 & 0 & -1 & 1 \\
\hline 1 & 1 & 1 & 0 & 1 \\
\hline
\end{tabular}

Table 4 summarizes noise margins corresponding to PTI and NTI, respectively. It can be seen from the Table 4 that significant improvement in noise margins in PTI and NTI is observed over the corresponding designs in Ref. 11.

Table 5 summarizes simulated rise time $\left(t_{r}\right)$, fall time $\left(t_{f}\right)$ of PTI and NTI, respectively, and propagation delay times $\left(\mathrm{t}_{\mathrm{pLH}}, \mathrm{t}_{\mathrm{pHL}}\right)$ of ternary full adder circuit. The simulated transient behavior of these circuits are compared with corresponding circuits implemented in DECMOS technology [11] for $0 \mathrm{pF}$ and $15 \mathrm{pF}$ equivalent load capacitance, $C_{L}$ and unbuffered circuit conditions. It can be seen from Table 5 that the rise and fall times of PTI shows an improvement by a factor of 14 and 4 and that of NTI by a factor of nearly 4 and 17, respectively over that of earlier designs implemented in DECMOS technology. It is also seen in Table 5 that the present full adder design performs better than the counterpart DECMOS design.

The fabricated device was tested for its performance evaluation and meets the required logic levels of ternary full adder summarized in Table 1. The PTI and NTI circuits were tested under pulse transient conditions with an equivalent $15 \mathrm{pF}$ load capacitance, $C_{L}$ and compared with the corresponding simulations. The results are summarized in Table 5 for $C_{L}=15 \mathrm{pF}$. The $15 \mathrm{pF}$ load capacitance corresponds to a $15 \mathrm{pF}$ input capacitance to the TEK 2467B oscilloscope used in the measurement which acts as a load to the device under test. It is seen from Table 5 that the measured values are in good agreement with the corresponding values obtained from simulations.

The power dissipation calculated from SPICE is summarized in Table 6 for PTI, NTI and ternary full adder, respectively. It is noticed that both designs in the present work and Ref. 11 exhibit power consumption in the microwatt power range. It is worth mentioning that the present design uses nearly one half of the silicon area of Ref. 11 for the design of a ternary full adder circuit.

\section{CONCLUSIONS}

A ternary full adder has been designed using fourteen T-gates and implemented in MOSIS two micron CMOS n-well process. The T-gate uses a $J_{k}$ arithmetic circuit and three ternary switches. The $J_{k}$ arithmetic circuit mainly consists of PTI and NTI apart from NOR, inverter and buffer circuits.

The PTI and NTI have been designed using an inverter and pass-transistors at its output. The design of PTI and NTI is fully compatible with the MOSIS two micron CMOS n-well process. It is shown that the performance 
TABLE 2

Averaged SPICE NMOS model parameters

\begin{tabular}{lll}
\hline & \multicolumn{1}{c}{ NMOS Parameters } \\
\hline LD $=0.24974 \mathrm{U}$ & TOX $=421.00001 \mathrm{E}-10$ & NSUB $=2.296064 \mathrm{E} 16$ \\
VTO $=0.94$ & KP $=5.504 \mathrm{E}-5$ & GAMMA $=0.9961$ \\
PHI $=0.6$ & UO $=628.787$ & UEXP $=0.22018$ \\
UCRIT $=115298$ & DELTA $=1.041739 \mathrm{E}-5$ & VMAX $=83151.5$ \\
XJ $=0.25 \mathrm{U}$ & LAMBDA $=1.67204 \mathrm{E}-2$ & NFS $=2.509221 \mathrm{E} 12$ \\
$\mathrm{NEFF}=1$ & NSS $=1 \mathrm{E} 10$ & TPG $=1$ \\
RSH $=27.36$ & CGDO $=3.283127 \mathrm{E}-10$ & CGSO $=3.28322 \mathrm{E}-10$ \\
CGBO $=4.96808 \mathrm{E}-10$ & $\mathrm{CJ}=4.1066 \mathrm{E}-4$ & MJ $=0.467277$ \\
CJSW $=3.9772 \mathrm{E}-10$ & MJSW $=0.334688$ & PB $=0.8$ \\
\hline
\end{tabular}

TABLE 3

Averaged SPICE PMOS model parameters

\begin{tabular}{lll}
\hline & \multicolumn{1}{c}{ PMOS Parameters } \\
\hline LD $=0.25000 \mathrm{U}$ & TOX $=421.00001 \mathrm{E}-10$ & NSUB $=5.917000 \mathrm{E} 15$ \\
VTO $=0.96$ & KP $=2.296 \mathrm{E}-5$ & GAMMA $=0.5057$ \\
PHI $=0.6$ & UO $=262.000$ & UEXP $=0.22505$ \\
$\mathrm{UCRIT}=21136.5$ & DELTA $=.721685$ & VMAX $=41563.6$ \\
XJ $=0.25 \mathrm{U}$ & LAMBDA $=5.597 \mathrm{E}-2$ & NFS $=8.0389 \mathrm{E} 11$ \\
NEFF $=1.001$ & NSS $=1 \mathrm{E} 10$ & TPG $=1$ \\
RSH $=70.00$ & CGDO $=3.286622 \mathrm{E}-10$ & CGSO $=3.28322 \mathrm{E}-10$ \\
CGBO $=4.75120 \mathrm{E}-10$ & CJ $=12.0692 \mathrm{E}-4$ & MJ $=0.431872$ \\
CJSW $=1.9981 \mathrm{E}-10$ & MJSW $=0.177313$ & PB $=0.7$ \\
\hline
\end{tabular}

of PTI, NTI and ternary full adder implemented in CMOS technology closely matches with designs implemented in corresponding DECMOS technology. There is very good agreement between simulated and measured voltage transfer characteristics, noise margins and transient times for PTI, NTI and ternary full adder, respectively. A description of the design of ternary NOR, ternary NAND and simple ternary inverter without using depletion mode transistors and resistors are also included for completeness.

In the low power design range, the present design of ternary circuits uses lesser number of components and thereby reducing the chip area to nearly one half compared to designs of DECMOS technology. Furthermore,

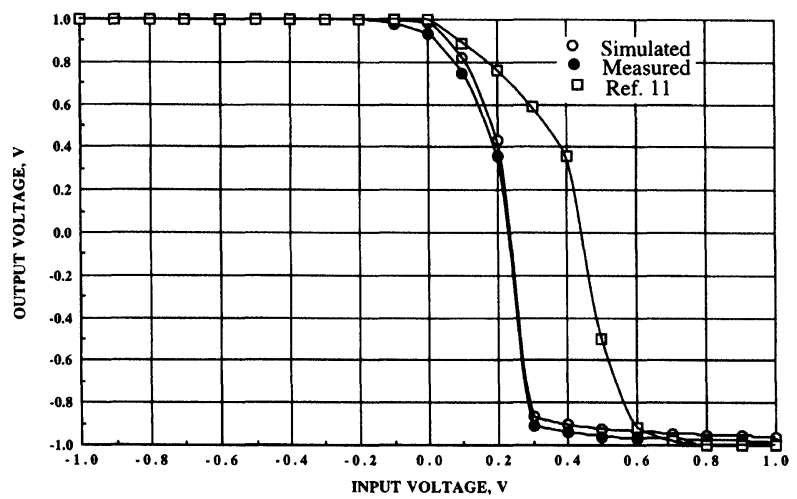

FIGURE 9 (a). Voltage transfer characteristics of a PTI. the use of depletion mode devices in the present work has been eliminated.

In the present work, the design of ternary full adder and its building blocks are designed within the limitation of the MOSIS foundry for the fabrication such as the non-availability of process modification to vary threshold voltages of MOSFETS. However, the present design could be further improved with the flexibility in process modification.

\section{Acknowledgements}

Authors are very grateful to the reviewers for their valuable comments and suggestions.

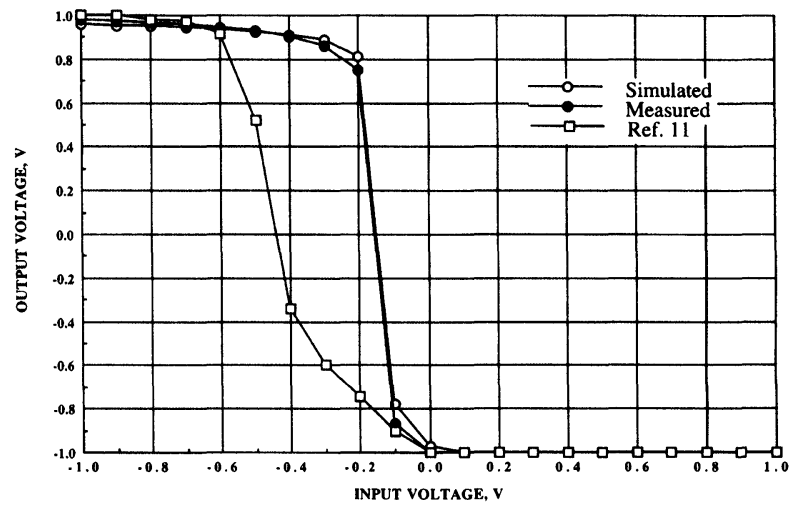

FIGURE 9 (b). Voltage transfer characteristics of a NTI. 
TABLE 4

Noise margins characteristics

\begin{tabular}{llcc}
\hline \multirow{2}{*}{ Gate } & \multicolumn{2}{c}{ Noise Margin, Volts } \\
\cline { 3 - 4 } & & $1--1$ & $-1-1$ \\
\hline \multirow{3}{*}{ PTI } & Present Work & 0.10 & 0.70 \\
& Measured & 0.10 & 0.70 \\
& Ref. 11 & 0.10 & 0.45 \\
\multirow{3}{*}{ NTI } & Present Work & 0.80 & 0.10 \\
& Measured & 0.80 & 0.10 \\
& Ref. 11 & 0.45 & 0.10 \\
\hline
\end{tabular}

TABLE 5

Transient times

\begin{tabular}{llcc}
\hline Gate & Load $\left(C_{L}, \mathrm{pF}\right)$ & $\begin{array}{c}t_{r}, \mathrm{~ns}(10-90 \%) \\
-1-1\end{array}$ & $\begin{array}{c}t_{f}, \mathrm{~ns}(90-10 \%) \\
1--1\end{array}$ \\
\hline PTI & 0 (Sim) & 5 & 3 \\
& 0 (Ref. 11) & 70 & 13 \\
& 15 (Sim) & 405 & 415 \\
& 15 (Meas) & 400 & 417 \\
NTI & 0 (Sim) & 4 & 4 \\
& 0 (Ref. 11) & 15 & 70 \\
& 15 (Sim) & 416 & 210 \\
& 15 (Meas) & 426 & 223 \\
Ternary & 0 (Sim) & $15^{*}$ & $22^{* *}$ \\
Full & 0 (Ref. 11) & $50^{*}$ & $83 * *$ \\
Adder & 15 (Sim) & 474 & 739 \\
& 15 (Meas) & 458 & 714 \\
\hline
\end{tabular}

$*_{\mathrm{pLH}}$
$* * \mathrm{t}_{\mathrm{pLH}}$

TABLE 6

Power dissipation

\begin{tabular}{lcc}
\hline \multirow{2}{*}{ Gate } & \multicolumn{2}{c}{ Power dissipation } \\
\cline { 2 - 3 } & Present work & Ref. 11 \\
\hline PTI & $0.8 \mathrm{nW}$ & $1.97 \mu \mathrm{mW}$ \\
NTI & $12 \mu \mathrm{W}$ & $29 \mathrm{nW}$ \\
Ternary Full Adder & $15 \mu \mathrm{W}$ & $0.14 \mu \mathrm{W}$ \\
\hline
\end{tabular}

*Power dissipation obtained using SPICE model parameters of Tables 2 and 3.

\section{References}

[1] J.T. Butler, Multiple-Valued Logic in VLSI, IEEE Computer Society Press Technology Series, Los Alamitos, California, 1991.

[2] A.K. Jain, M.H. Abd-El-Barr and R.J. Bolton, "A new structure for CMOS realization of MVL functions," International Journal of Electronics, vol. 74, no. 2, pp, 251-263, 1993.
[3] S.L. Hurst, "Two decades of multiple valued logic-an invited tutorial," in Proceedings of IEEE International Symposium on Multiple-Valued Logic, p. 164, May 1988.

[4] S.L. Hurst, "Multiple-valued logic-its status and its future," IEEE Transactions on Computers, vol. C-33, no. 12, pp. 1160-1179, December 1984.

[5] H.T. Mouftah and I.B. Jordan, "Integrated circuits for ternary logic," in Proc. ISMVL-74, (Morgantown, WV), pp. 285-302, May 1974.

[6] H.T. Mouftah and I.B. Jordan, "Design of ternary COS/MOS memory and sequential circuits," IEEE Trans. Computers, vol. C-26, pp. 281-288, March 1977.

[7] H.T. Mouftah, "A study on the implementation of three-valued logic," in Proc. ISMVL-76, (Bloomington, IL), pp. 123-126, May 1976.

[8] J.M. Carmona, J.L. Huertas, and J.I. Acha, "Realization of three-valued C.M.O.S. cycling gates," Electron. Lett., vol. 14, pp. 288-290, 1978.

[9] H.T. Koanantakool, "Implementation of ternary identify cell using CMOS integrated circuits,” Electron. Lett., vol. 14, pp. 462-464, 1978.

[10] H.T. Mouftah and K.C. Smith, "Injected voltage low-power CMOS for 3-valued logic," IEE Proceedings, vol. 129, pt. G, no. 6, pp. 270-271, December 1982.

[11] A. Heung and H.T. Mouftah, "Depletion/enhancement CMOS for a low power family of three-valued logic circuits," IEEE Journal of Solid-State Circuits, vol. SC-20, no. 2, pp. 609-615, April 1985.

[12] H.T. Mouftah and A.I. Garba, "VLSI implementation of a 5- trit full adder," IEE Proceedings, vol. 131, pt. G, pp. 214-220, October 1984.

[13] H.M. Razavi and S.E. Bou-Ghazale, "Design of a fast CMOS ternary adder," in Proceedings of IEEE International Symposium on Multiple-Valued Logic, p. 20, May 1987.

\section{Biographies}

A. SRIVASTAVA has served as a scientist at the Central Electronics Engineering Research Institute, Pilani; and on the faculty of Birla Institute of Technology and Science, Pilani, India; North Carolina State University; State University of New York; University of Cincinnati and as a UNESCO Fellow; as a visiting scientist and UNESCO Fellow at the University of Arizona. Currently he is an Associate Professor of Electrical and Computer Engineering at the Louisiana State University in Baton Rouge. His research interests include CMOS/BiCMOS VLSI design and device modeling, cryogenic CMOS electronics, smart gas sensors and MEMS. His e-mail address is ashok@gate.ee.lsu.edu.

K. VENKATAPATHY has graduated with a M.S. degree in Electrical Engineering from the Louisiana State University, Baton Rouge in 1994. $\mathrm{He}$ has received his B.E. (Hons.) degree in Electronics and Electrical Engineering from the Birla Institute of Technology and Science, Pilani in 1990. His research interests include multiple-valued logic VLSI design and low-temperature CMOS electronics. 

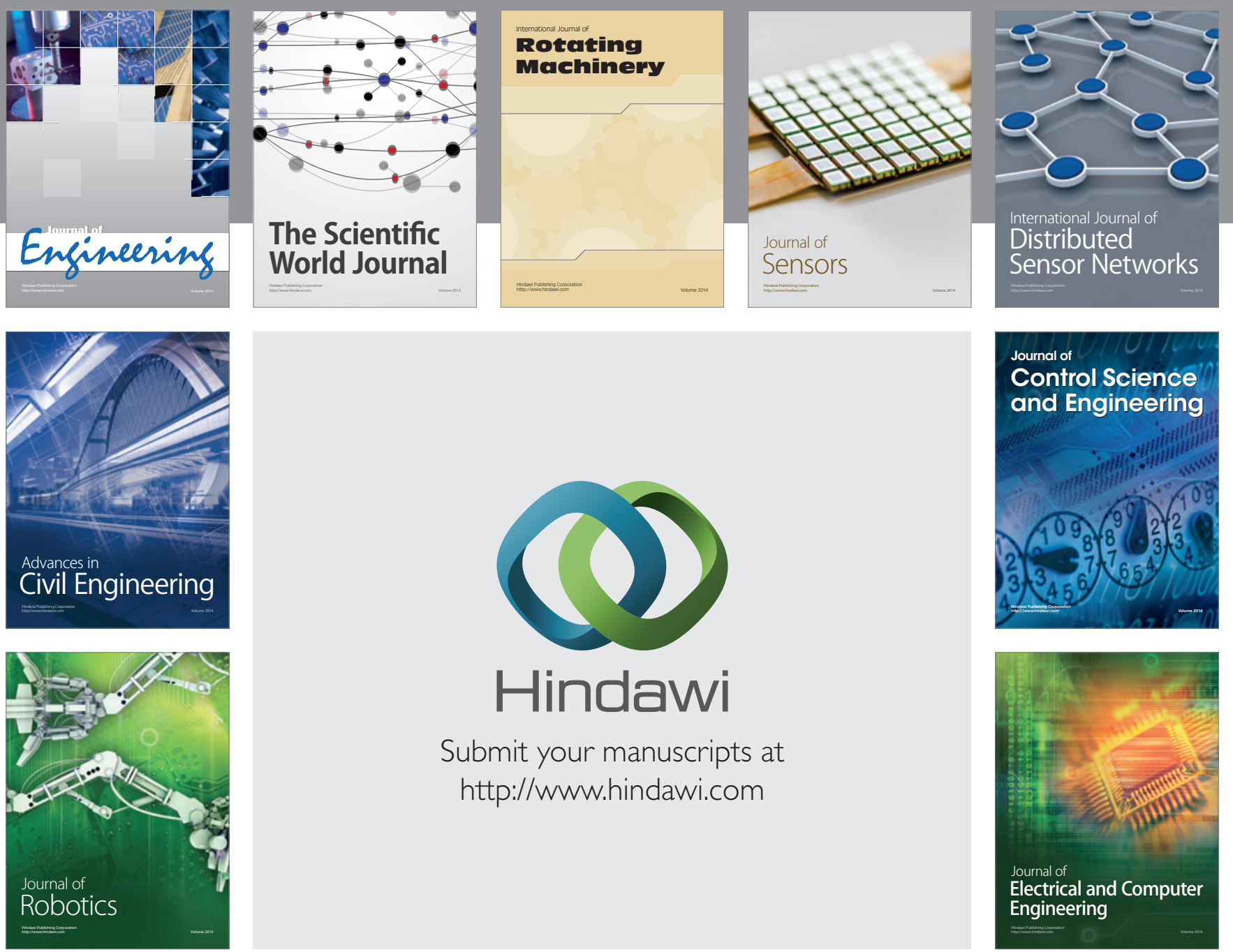

Submit your manuscripts at

http://www.hindawi.com
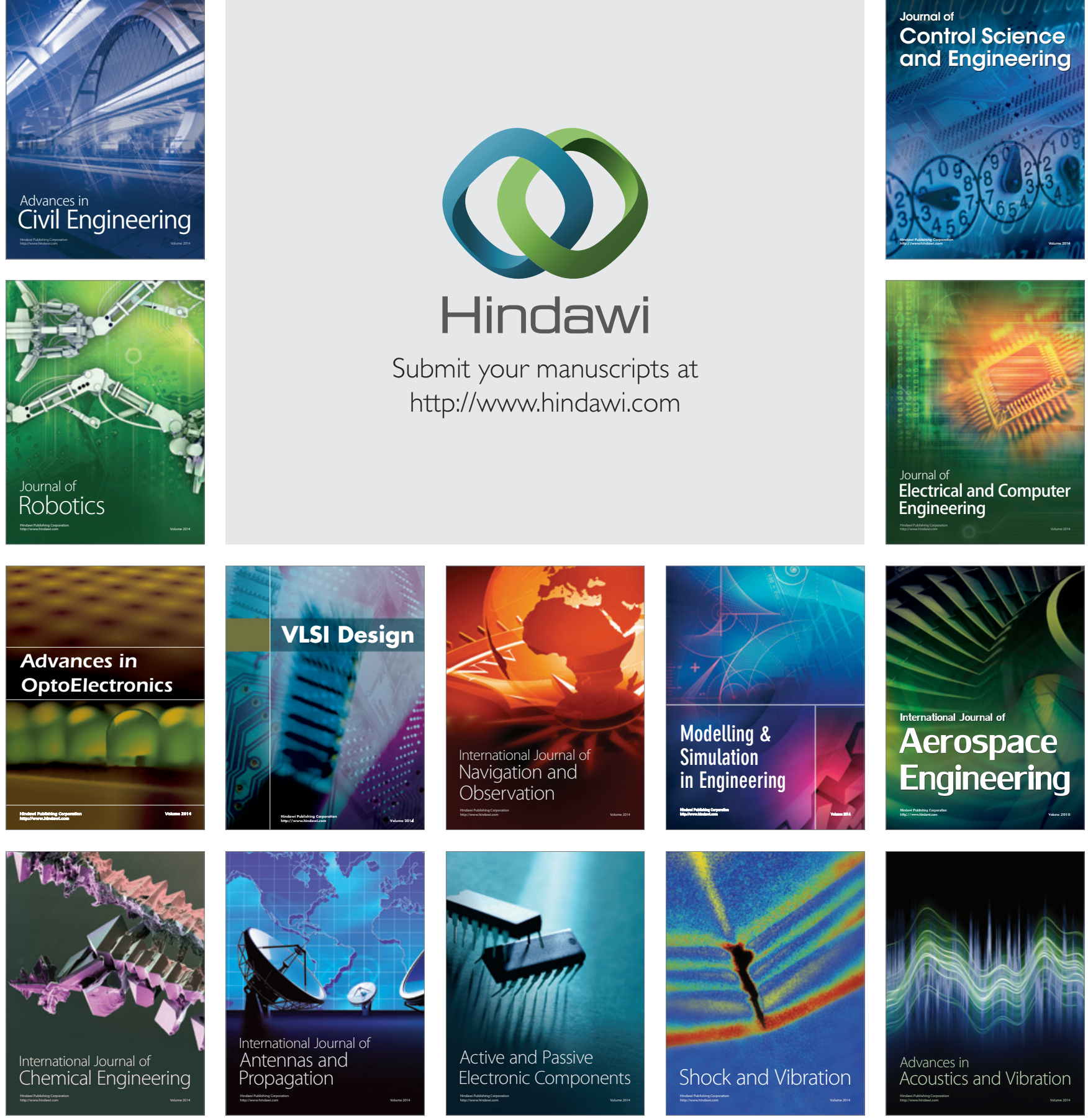